$16^{\text {th }}$ International Conference on

AEROSPACE SCIENCES \& AVIATION TECHNOLOGY,

ASAT - 16 - May 26 - 28, 2015, E-Mail: asat@ mtc.edu.eg

Military Technical College, Kobry Elkobbah, Cairo, Egypt

Tel : +(202) 24025292 - 24036138, Fax: +(202) 22621908

\title{
Vibration Isolation Control of Inertial navigation sensors Using Transfer Matrix Method for Multibody Systems
}

\author{
Hossam Hendy ${ }^{*}$, Xiaoting Rui ${ }^{* *}$, Mostafa Khalil ${ }^{*}$ and Zhou Qinbo ${ }^{* * *}$
}

\begin{abstract}
Inertial navigation sensors are widely used in space missions, orbit determination, and vehicle's navigation, guidance and control sections. These sensitive sensors can be affected by extreme vibration excitations; which must be protected from high level excitations with addition to accuracy improvement. Although Inertial Measurement Unit (IMU) singledegree-of-freedom (SDOF) model is the simplest model to study vibration isolation but a fortune of information may be obtained about isolation characteristics, while two-degree-offreedom (2DOF) IMU model is closer to real model. The new developed Transfer Matrix Method for Multibody Systems (MSTMM) compared with ordinary dynamic methods has modeling flexibility, low order system matrix, and high computational efficiency, without need of establishing the system's global dynamic equations. It has the merit of merging classical control techniques without needing especial treatments for different dynamics models. Main objectives are studying of flexibility effects in isolation design using MSTMM and transmissibility study, and then formulating a relationship with different control strategies. Simulated and experimental results indicated an availability of merging that allow wider developed control applications using MSTMM for more complex systems' models. Dilatation in studying controlled systems using MSTMM provides a possibility for incoming control applications because of the efficient dealing with the increased complexities of multirigid-flexible-body problems.
\end{abstract}

Keywords: Inertial sensors, Vibration analysis, MSTMM, PID controller, Transmissibility.

\section{Introduction:}

Different components and systems can be modeled as single-degree-of-freedom (SDOF) model, for example accelerometer model can be modeled as a base-excited mass-springdamper system [1-2], and also the Inertial Measurement Unit (IMU) which is commonly used for vibration isolation analysis can be analyzed as SDOF model. Although of its simplicity but quantifies many results of isolation specifications [3]. Its severe limitation is the noisy results when the base and/or equipment are flexible without vibration isolation/absorption systems.

\footnotetext{
*PhD., Military Technical College, Cairo, Egypt.

Professor, Institute of Launch Dynamics, Nanjing University of Science and Technology, China.

* PhD., Military Technical College, Cairo, Egypt.

*** $\mathrm{PhD}$. candidate, Institute of Launch Dynamics, Nanjing Univ. of Science and Technology, China.
} 
Flexibility effects were considered in previous researches but always been approximated leading to truncation errors [4]. Vibration control aims to mitigate undesired oscillations in a part of a system, it has two types as isolators and absorbers; vibration isolation occurs when resilient elements are connected between a vibrating part and a sensitive part, which called force transmissibility or source isolation if the elements are placed between the vibrating part and the surrounding structure, and called displacement transmissibility or receiver isolation if placed between the sensitive part and the surrounding structure [5-6], while vibration absorption occurs when adding a device to decay vibrations for the host system. Its main elements are mass, spring and damper elements; it is useful for controlling both narrow and broadband vibrations. Transmissibility ratio $\left(T_{r}\right)$ is a factor to characterize vibration transmission performance; it is a ratio of the measured transferred vibration level (displacement or force) for a part of a system to vibration excitation [7]. For the isolation field of research; transmissibility ratio is a beneficial indicator for isolator design acceptance. The system's vibration characteristics can be represented by a graph of transmissibility ratio in decibels $(\mathrm{dB})$ versus frequency in Hertz $(\mathrm{Hz})$. A general transmissibility curve can indicate its information such as damping ratio, natural frequency, and isolation efficiency.

\section{IMU Problem Formulation:}

The proposed fixation scenarios for a given IMU unit can be shown in figure 1. In figure (1a), the isolators are located on perpendicular surfaces, and the coordinates of the discrete isolators are considered such that the elastic center of the isolation system is close to the geometric center of the object.

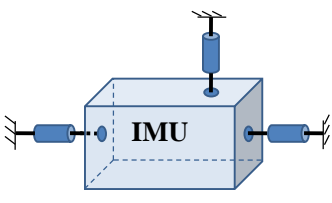

( a )

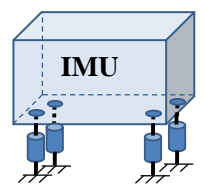

( b )

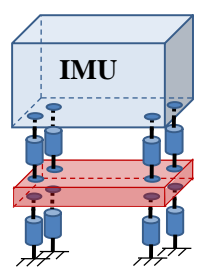

(c)

Fig. 1: Dynamic model of controlled IMU

Location adjustment is required in order to make cross transmissibility components as close to zero as possible among any two directions in order to make measurements correct because controlling not only the response amplitude is important for the design of an IMU but also the response characteristic in each direction. Response of the system in angular directions to linear acceleration should be applied as low as possible in order to get exact measurements of angular velocity and angular acceleration from gyroscopes. So, the cross stiffness components between translational and angular directions should be as close to zero as possible. This method is difficult to be implemented due to that any small difference or shift from the center of the object will add more forces and moments to the system disturbances [8]. In figure 1-b, both isolated system and foundation base are assumed rigid neglecting base mass relative to IMU mass. Firstly and for simplicity purposes of vibration test using vibration shaker, displacements of isolators' inputs in vertical direction are considered as same amplitude; 
yields to a simplified IMU single-degree-of-freedom (SDOF) model as shown in figure 2 . Although it is a very simple model but a fortune of information may be obtained about isolation characteristics. For design purposes, the isolator is massless and modeled as a viscous damper with coefficient $\mathrm{c}$ and spring with stiffness $\mathrm{k}$, with assuming that they are constant in the frequency range of interest [9-11]. The values for the isolator's spring and damper are chosen to reduce as much vibration in the system as possible.

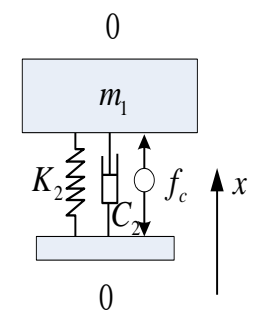

Fig. 2 SDOF assembly model

According to figure 2 and MSTMM basics, the direction of $x$ axis indicates the positive direction, $\boldsymbol{Z}_{i, j}=\left[X, Q_{x}\right]_{, j}$ is the state vector of an arbitrary connection point of the system, where $i$ and $j$ are the sequence numbers of body and hinge elements, respectively [12]. Considering the vibration excitation input from a moving structure, the boundary end from 0 to element 2 is assumed to be in the form of $x_{2,0}=A e^{i \mathrm{Wt}}$, where $A$ is the complex amplitude and $\mathrm{W}$ is the movement frequency. Thus, in a steady-state sense, the whole system experiences the forced vibration in the sine form [13-14]. The lumped mass transfer equation is:

$$
\boldsymbol{Z}_{1, O}=\boldsymbol{U}_{1} \boldsymbol{Z}_{1, I}
$$

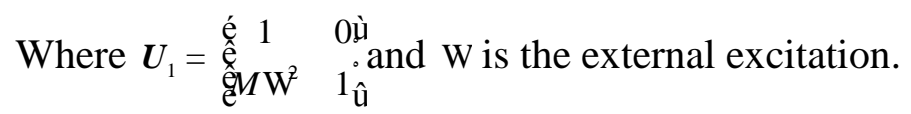

From figure 2, the transfer equation of the controlled element is:

$$
\boldsymbol{Z}_{2, O}=\boldsymbol{U}_{2} \boldsymbol{Z}_{2, I}+\boldsymbol{E}_{c} F_{c}
$$

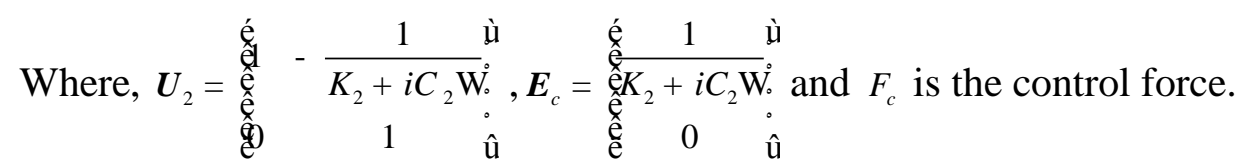

Considering a negative feedback PID controller and $x_{1,0}$ as the measurement signal, the control force takes the form:

$$
f_{c}=-\varepsilon_{e} k_{P} x_{1,0}+k_{I}=0
$$

Where $k_{P}, k_{I}$, and $k_{D}$ are proportional, integral, and differential gains respectively. Applying the transformation of $x=X e^{i \mathrm{Wt}}, f_{c}=F_{c} e^{i \mathrm{~W} t}$ yield:

$$
F_{c}=-\underset{\varepsilon}{\varepsilon} k_{P}+\frac{k_{I}}{i \mathrm{~W}}+i \mathrm{~W} k_{D} \dot{\overline{\bar{i}}} X_{1,0}^{9}=:-C(i \mathrm{~W}) X_{1,0}
$$

Meanwhile 


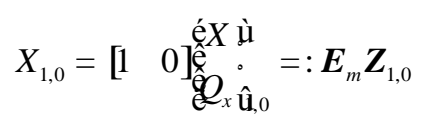

The controlled system structure diagram [15 - 16] can be configured as shown in Fig. 3.

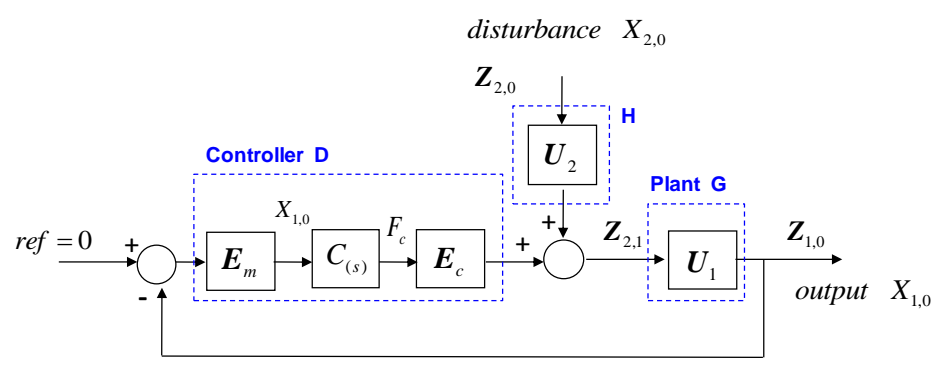

Fig. 3: The structure diagram of SDOF controlled system

From figure 3, the overall transfer equation of the controlled system is:

$$
\begin{gathered}
\boldsymbol{Z}_{1,0}=\boldsymbol{U}_{1}\left(\boldsymbol{U}_{2} \boldsymbol{Z}_{2,0}-\boldsymbol{E}_{c} C(i \mathrm{~W}) \boldsymbol{E}_{m} \boldsymbol{Z}_{1,0}\right) \\
\mathrm{W} \boldsymbol{Z}_{2,0} \quad\left(\boldsymbol{U}_{1} \boldsymbol{U}_{2}\right)^{-1} \dot{d}_{2} \quad C\left(i+\text { 建 } \boldsymbol{E}_{c} \boldsymbol{E}_{m} \grave{\mathrm{u}} \boldsymbol{Z}_{1,0}\right.
\end{gathered}
$$

Considering boundary conditions of $\boldsymbol{Z}_{1,0}=\left[\begin{array}{ll}X & 0\end{array}\right]_{, 0}$, and expressing $\boldsymbol{Z}_{2,0}=\left[\begin{array}{ll}A & Q_{x}\end{array}\right]_{2,0}$ yield:

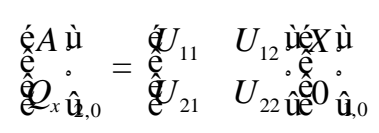

Thus the frequency response function from the disturbance input to the output is:

$$
\frac{X_{1,0}}{A}=\frac{1}{U_{11}}
$$

From transmissibility ratio definition indicated in equation 9; setting $k_{P}=k_{I}=k_{D}=0$ acquires a passive transmissibility formula and/or the uncontrolled system transfer function. While any other values for controller gains indicates an active transmissibility for the controlled system. Expanding equation 9 without control force yields:

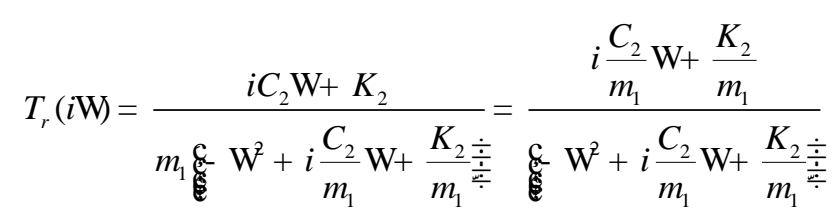

From control theories; the $2^{\text {nd }}$ order standard transfer function transmissibility ratio can be written as: 


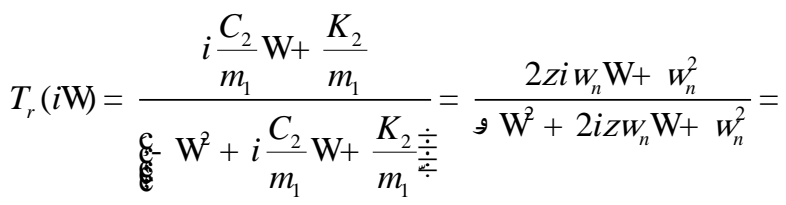

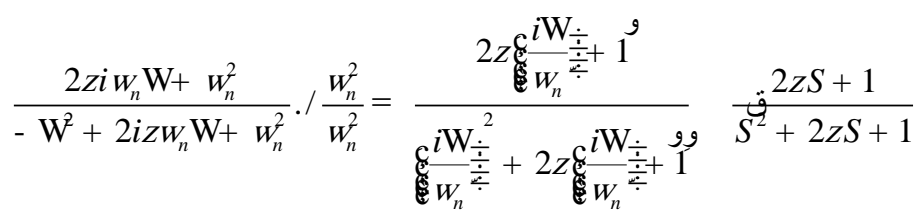

Considering different values for the damping coefficient $z$ give different efficiencies for transmissibility ratios, these curves can be shown in figure 4 .

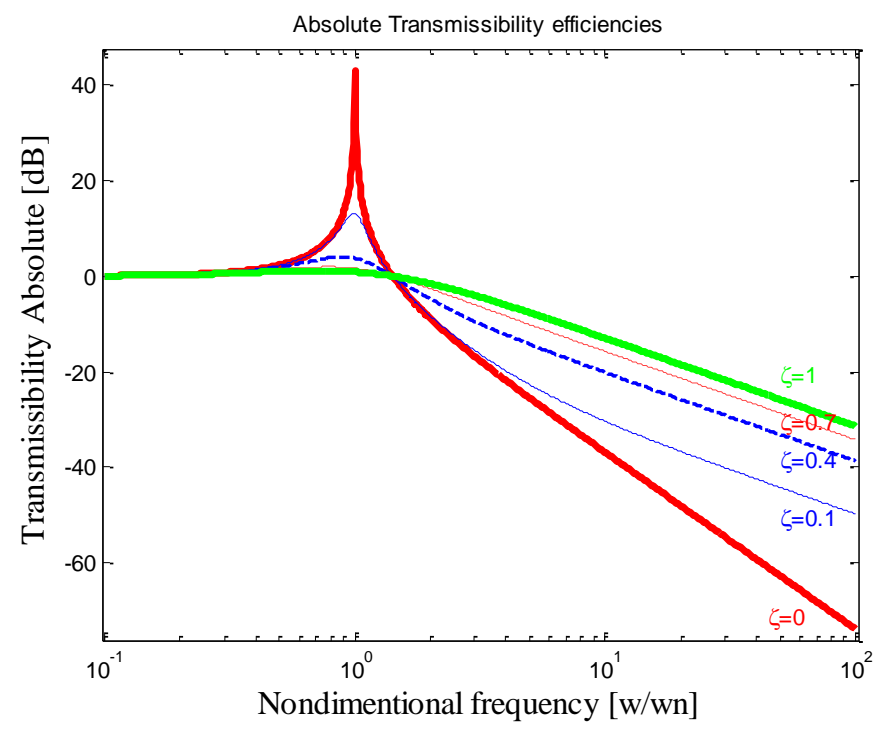

Fig. 4: SDOF nondimensional transmissibility

From figure 4, it's obvious that the transmissibility ratio due the spring-mass-damper (passive isolator) is unbounded at the natural frequency of the system when no damping is present but the transmissibility is decreased at high frequency regions, in the other side, increasing the damping ratio decreases the transmissibility at the natural frequency but amplifies it at high frequencies. The challenge is to quantify the unbounded transmissibility at the natural frequency with keeping on the low transmissibility for the high frequencies.

So, active control can alleviate that by attenuating transmissibility amplification at high frequencies while bounding it at the natural frequency. Also, it is obvious that the extreme amplitude occurs when no damping is present, or can call it as the isolator has no damper. Considering the control scheme configured in figure 3, with the assumptions of no damping $(z=0)$, transmissibility ratio derived in equation (11) can be rewritten as:

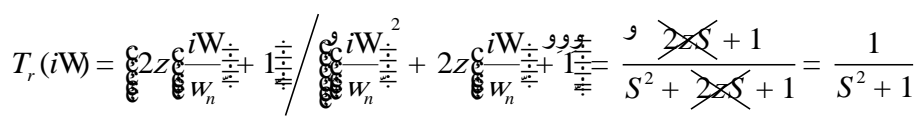

In case of present of active control isolation, the control law schematic diagram in figure 3 can be redrawn and then rearranged to match the classical control schemes as: 


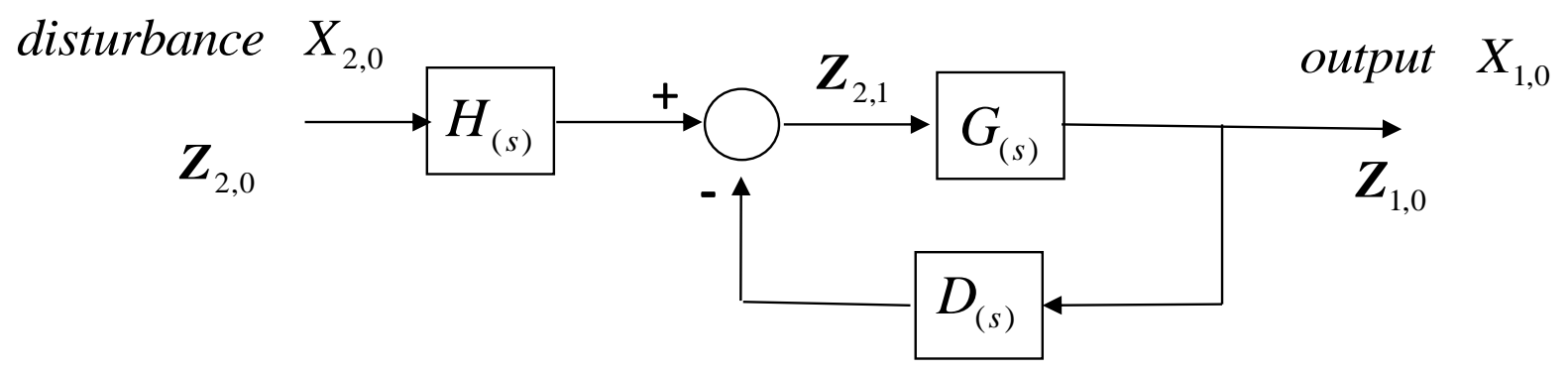

Fig. 5: The proposed model control schematic diagram

The new transfer function of the system with absence of damping and at the resonant condition is deduced in equation 12; applying Routh stability test and root locus criteria, the system is critically stable, so the active control law should provide a kind of damping to the model.

For increasing the reality of studying system the proposed model shown in figure 1-b can be expanded as shown in figure 1-c where the rigid foundation can be replaced by a massspring-damper system which will represent the base flexibility and transforms the model to be 2DOF model as shown in figure 6.

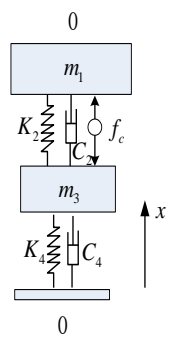

Fig. 6: The proposed model as 2DOF model

The boundary end $x_{4,0}$ is assumed as $x_{4,0}=A e^{i \mathrm{Wt}}$, with complex amplitude $A$ and frequency w. Current section highlights one of the most important advantages of MSTMM when dealing with higher order systems, higher complexities and structures that can use the previous deduced SDOF model to combine new transfer matrices for additional elements with simple treatment as follow:

New transfer matrices $\mathrm{U}_{3}$ and $\mathrm{U}_{4}$ due new additional elements of $\mathrm{m}_{3}, \mathrm{~K}_{4}$, and $\mathrm{C}_{4}$ are added as series connection without need to change dynamical equations as cases of using classical methods (Newton, Lagrange, ...etc.). The required change will appear when considering control schematic diagram shown in figure 5 to be matched with the new $2 \mathrm{DOF}$ model as:

1- For SDOF model; the block $\mathrm{H}_{(\mathrm{s})}=\mathrm{U}_{2}$

2- For 2DOF model; $\mathrm{H}_{(\mathrm{s})}=\mathrm{U}_{4} \mathrm{U}_{3} \mathrm{U}_{2}$

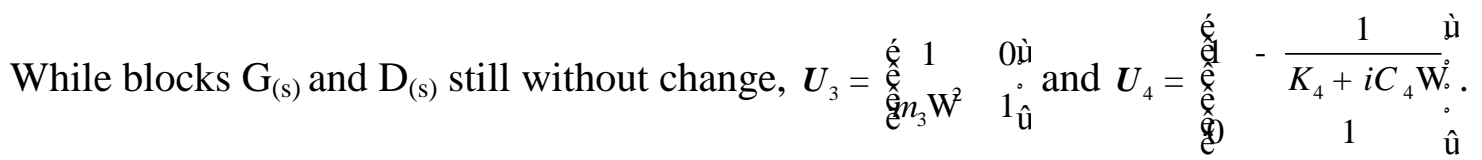




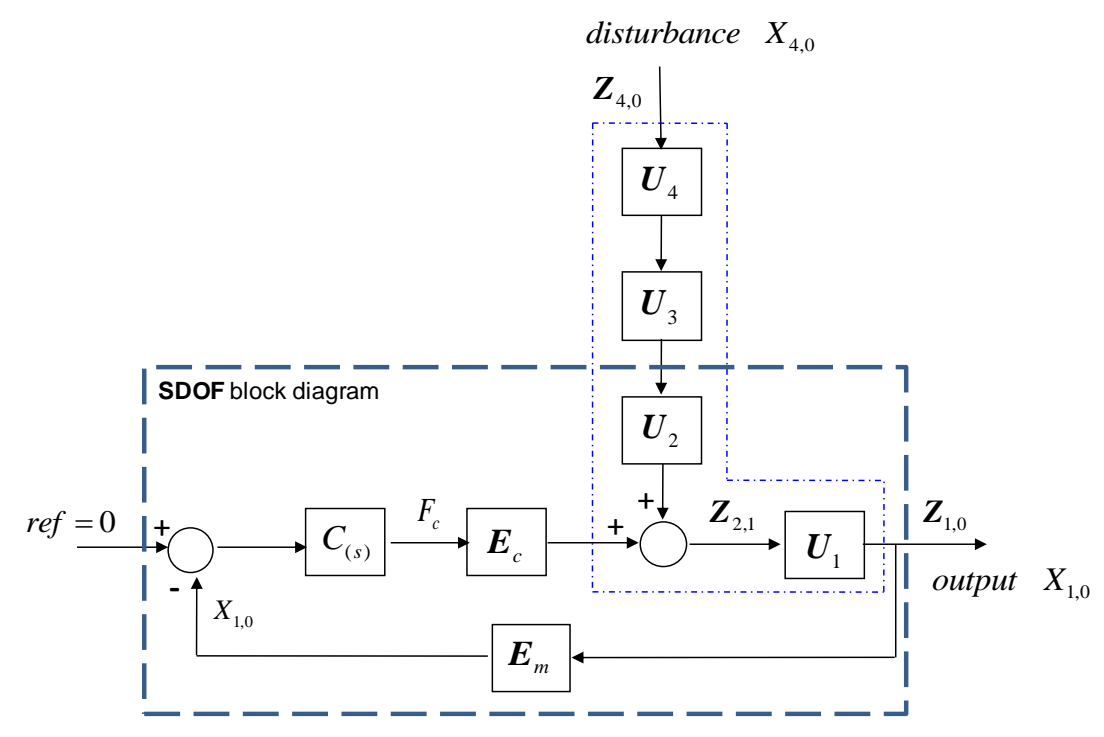

Fig. 7: The structure diagram of 2DOF controlled system

From the schematic diagram shown in figure 7 ; the total transfer equation of $2 \mathrm{DOF}$ controlled system is:

$$
\boldsymbol{Z}_{4,0}=\left(\boldsymbol{U}_{1} \boldsymbol{U}_{2} \boldsymbol{U}_{3} \boldsymbol{U}_{4}\right)^{-1} \dot{d}_{2}+C(i \mathrm{~W}) \boldsymbol{U}_{1} \boldsymbol{E}_{c} \boldsymbol{E}_{m} \text { ù } Z_{1,0}
$$

Considering the boundary condition $\boldsymbol{Z}_{1,0}=\left[\begin{array}{ll}X & 0\end{array}\right]_{, 0}$, and expressing $\boldsymbol{Z}_{4,0}=\left[\begin{array}{ll}A & Q_{x}\end{array}\right]_{4,0}$ yield:

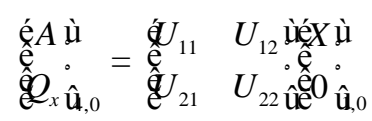

The transfer function from the disturbance input to the output is:

$$
\frac{X_{1,0}}{A}=\frac{1}{U_{11}}
$$

Similar to equation 9; the transmissibility ratio for both controlled and uncontrolled model can be deduced from equation 15 ; it can be expanded to be:

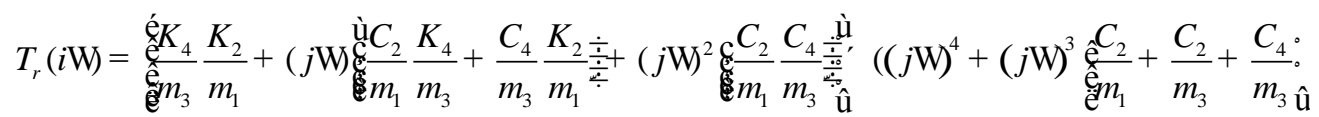

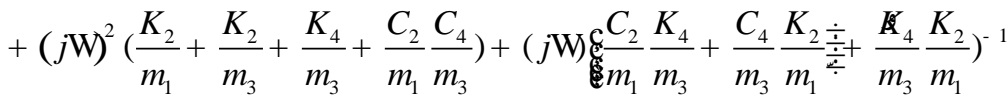

As general definition of the $2^{\text {nd }}$ order standard transfer function and from the related MSTMM model, the following quantities can be defined as:

$$
2 Z_{j} W_{n_{j}}=\frac{C_{2 j}}{m_{2 j-1}}, \quad W_{n_{j}}=\sqrt{\frac{K_{2 j}}{m_{2 j-1}}}, j=1,2
$$

Applying the previous definitions, get: 


$$
\begin{aligned}
& T_{R}=\left(W_{n_{2}}^{2} W_{n_{1}}^{2}+(j \mathrm{~W})\left(2 Z_{1} W_{n_{1}} W_{n_{2}}^{2}+2 Z_{2} W_{n_{2}} W_{n_{1}}^{2}\right)+\left(j \mathrm{~W}^{2}\left(4 Z_{1} Z_{2} W_{n_{1}} W_{n_{2}}\right)\right)^{\prime}\left((j \mathrm{~W})^{4}+(j \mathrm{~W})^{3}\left(2 Z_{1} W_{n_{1}}+2 Z_{1} W_{n_{1}} \frac{m_{1}}{m_{3}}+2 Z_{2} W_{n_{2}}\right)\right.\right. \\
& +(j \mathrm{~W})^{2}\left(W_{n_{1}}^{2}+W_{n_{1}}^{2} \frac{m_{1}}{m_{3}}+W_{n_{2}}^{2}+4 Z_{1} Z_{2} W_{n_{1}}^{2} W_{n_{2}}^{2}\right)+\left(j \mathrm{~W}\left(2 Z_{1} W_{n_{1}} W_{n_{2}}^{2}+2 Z_{2} W_{n_{2}} W_{n_{1}}^{2}\right)+W_{n_{2}}^{2} W_{n_{1}}^{2}\right)^{-1}
\end{aligned}
$$

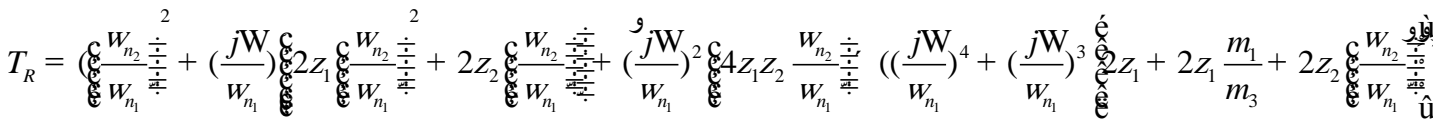

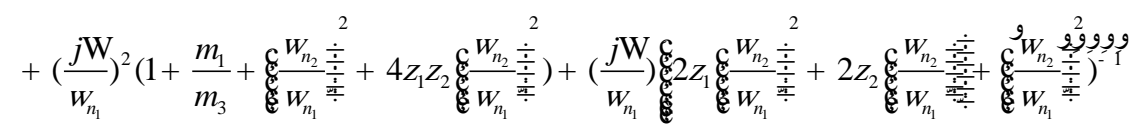

For the non dimensional analysis, considering $S=\frac{j \mathrm{~W}}{W_{n_{1}}}, \quad b=\frac{W_{n_{2}}}{W_{n_{1}}}, M=\frac{m_{1}}{m_{3}} ;$ yields:

$$
\begin{aligned}
T_{R}=\left\{b^{2}+\right. & \left.S\left(2 z_{1} b^{2}+2 z_{2} b\right)+S^{2}\left(4 z_{1} z_{2} b\right)\right\}\left\{S^{4}+S^{3}\left[2 z_{1}+2 z_{1} M+2 z_{2} b\right]\right. \\
+ & \left.S^{2}\left(1+M+b^{2}+4 Z_{1} z_{2} b^{2}\right)+S\left(2 z_{1} b^{2}+2 z_{2} b\right)+b^{2}\right\}^{-1}
\end{aligned}
$$

Considering $z_{1}=h z_{2}$ :

$$
\begin{array}{r}
T_{R}=\left\{b\left(2 h z_{2} S+1\right)\left(2 z_{2} S+b\right)\right\}^{\prime}\left\{S^{4}+S^{3}\left[2 h z_{2}+2 h z_{2} M+2 z_{2} b\right]\right. \\
\left.+S^{2}\left(1+M+b^{2}+4 h z_{2}^{2} b^{2}\right)+S\left(2 h z_{2} b^{2}+2 z_{2} b\right)+b^{2}\right\}^{-1}
\end{array}
$$

The transmissibility performance can by analyzed by changing the ratios between elements, and then an optimized technique can be applied to get the best results.

\section{Simulation, Experimental tests, and Results:}

Assuming the mass of isolation system is up to $1 / 4$ of the isolated system mass, and studying the system at the critical situation of no damping with equal spring's stiffness, the non dimensional frequency response for the absolute transmissibility after applying PID active control model can be drawn as shown in figure 8 .

The control law can be designed in time or frequency domains, the frequency domain design is preferred due to that MSTMM deals with dynamical models in frequency domain in simple ways, also the transmissibility can be quantified easier in frequency domain. 


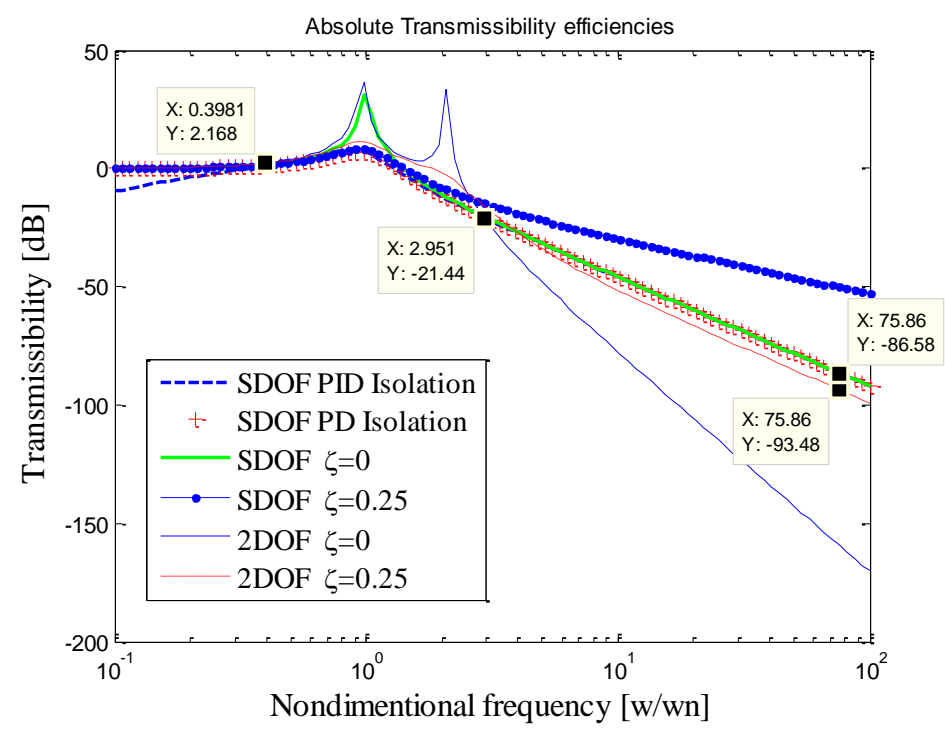

Fig. 8: The diagram of

Figure 8 illustrates effects of the applied PD and PID controllers, where the PD controller transfer function as nondimensional frequency is $D_{(s)}=K_{D} S+K_{P}$, the derivative gain $K_{D}=0.5$ can considered as a damping element value, and the proportional gain $K_{P}=0.01 \mathrm{can}$ considered as a stiffness element value. The PID controller nondimensional frequency transfer function is $D_{(s)}=K_{D} S+K_{P}+K_{I} / S$ with $K_{D}=0.75, K_{P}=0.01$, and $K_{I}=0.2$. Also damping ratios were changed to illustrate the damping ratio changing effects; results of figure 8 declared that at low frequency region before the resonant frequency, the transmissibility performance for passive isolation systems are the same although different damping values were used. For active isolations, the mean objective is to get best transmissibility performance especially at the resonance frequency.

From figure 8, it is obvious that PD controller performance has same performance at both lower and higher frequencies with overcoming the transmissibility unbounded problem at the resonance frequency [15-16]. But for PID controller; it was obvious that it has same performance as PD controller with better performance at lower frequency region, natural frequencies for both PD and PID controllers were small due to the value $K_{P}$ which assigned very small to limit the varying of the natural frequency shift with respect to the original system's natural frequency 


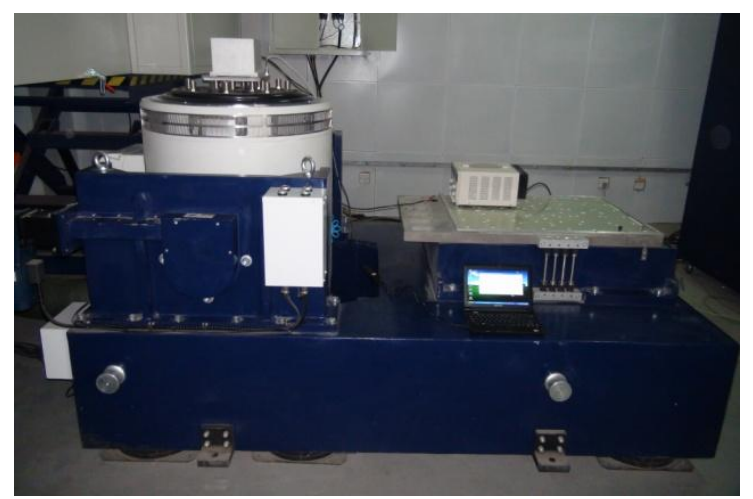

Fig. 9: Vibration shaker test bench

Figure 9 illustrates the vibration test using vibration shaker for an IMU module using two acceleration sensors for detecting input and output response signals; according to MILHDBK-340A and MIL-STD-1540C which illustrated that the induced random vibration for part of structure is due to the direct or indirect actions of excitations from acoustic, aerodynamic forces, burning processes, and random disturbances. These random vibrations can be expressed by the power spectral density (PSD) or as an acceleration spectral density in $\mathrm{g}^{2} / \mathrm{Hz}$ over the frequency range of at least 20 to $2000 \mathrm{~Hz}$; in this work the range of frequencies is 50 to $2000 \mathrm{~Hz}$ as configured at figure 10 .

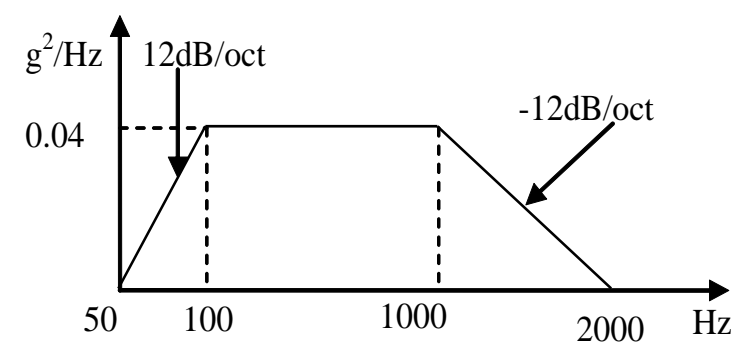

Fig. 10: Vibration shaker test input PSD

A rocket vehicle clearly experiences nonstationary vibration during its powered flight. A sample acceleration time history for the input excitation and the IMU passive dynamical response is shown in figure 11.

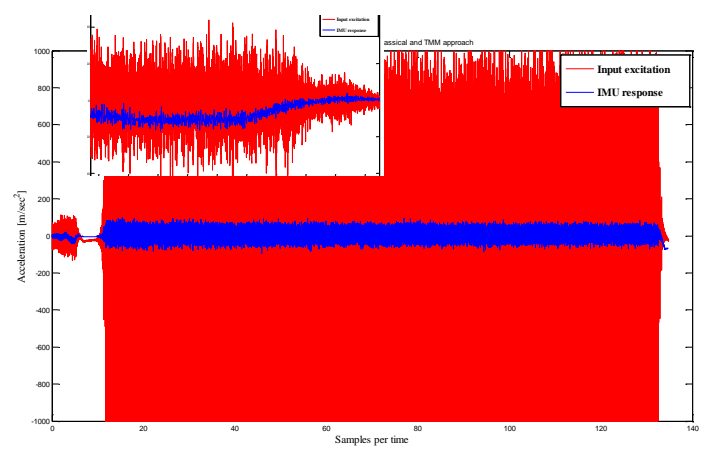

Fig. 11: Input and IMU output response 
The input excitation overall level calculated using the data of figure 11 is 7.6509 GRMS while the square root of the area under the curve of figure 10 is 7.6442 GRMS. Both values are equal, ignoring the small numerical error.

Figure 11 shows acceleration time history for input and accelerometer's measured values, which give many sample rates that will be difficult to be processed easily. The accelerometer's measurements curve is processed to give the standard deviation time history calculated every 0.2 second interval to minimize sample numbers and get faster calculations as shown in Figure 12.

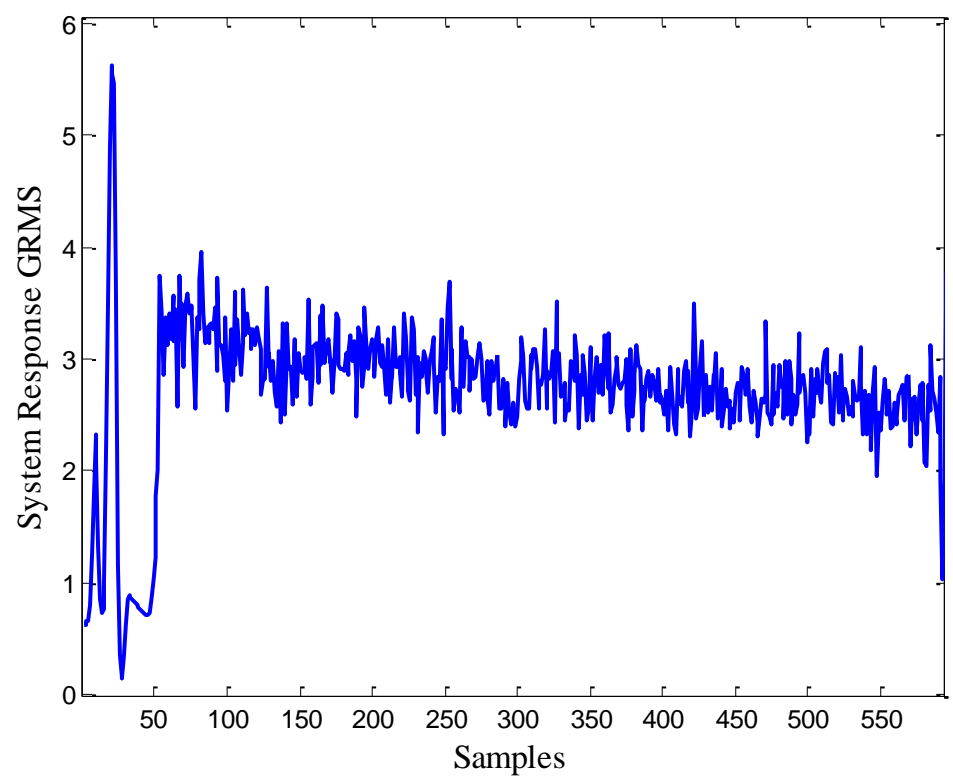

Fig. 12: system output response

Several reasons are exist for computing standard deviation time history such as:

1. The instantaneous time history may have over 1000,000 data samples which make it impractical to plotting these data for available computer hardware and graphing software, while the standard deviation can reduce these samples to few hundreds of samples, the standard deviation time history can be considered as data reduction tool.

2. It can be used to check if signal is stationary or nonstationary. From figure 12; it is clear that these data is nonstationary.

3. It can be used to declare sinusoidal signal if it is enveloped by random vibration due to that the sine function standard deviation is 0.707 times the peak value while the random vibration standard deviation is be 0.30 times the peak value.

The acceleration time history considered the input excitation data is simulated as a launched rocket vehicle responses due to motor ignition and launch acoustics vibration effects during first few seconds. In real case it should be decayed during 2-5 seconds but in current work and for purposes of testing IMU response; the test interval is expanded for longer time with controlling via shaker test bench. In real case, vibration levels become relatively in benign levels after few seconds that the rocket vehicle main vibration source is the motor 
burn. So; the considered acceleration levels overcome all expected levels after the interval of launching which has high levels of disturbances and acoustics effects.

From equation 11, Recall that the damping is often represented in terms of quality factor $Q=1 / 2 z$. In current experiment the IMU mass is $23 \mathrm{Kg}$ with natural frequency $f_{n}=97 \mathrm{~Hz}$. As mentioned before, the system transmissibility has critical response near zero damping ratio, so considering a ratio of 0.05 yields to $Q=10$, IMU output acceleration response related to Miles equation can thus be expressed as [3]:

$$
X_{1,0_{\text {GRMS }}}=\sqrt{\frac{p}{2} P f_{n} Q}
$$

While displacement response can be expressed as:

$$
X_{1,0_{\text {GRMS }}}=\frac{X_{1,0_{\text {GRMS }}}}{\left(2 p \cdot f_{n}\right)^{2}}
$$

Where $\mathrm{P}$ is the power spectral density level at $f_{n}$. IMU response at natural frequency is 7.04536 $\mathrm{G}_{\mathrm{RMS}}$ and displacement $2.8595 \mathrm{e}-5 \mathrm{G} . \mathrm{S}^{2}$.RMS; that can be transformed to be:

$$
X_{1,0}=2.8595 \mathrm{e}^{-5} \mathrm{G} \cdot \mathrm{s}^{2} \cdot \frac{386.09 \mathrm{in} / \mathrm{s}^{2}}{1 \mathrm{G}}=0.01104 \mathrm{in}_{\mathrm{RMS}}
$$

In current investigation, related results at $\mathrm{f}_{\mathrm{n}}=97 \mathrm{~Hz}$ are as follow: input power spectral density $\left(\mathrm{S}_{\mathrm{f}}\right)=.04\left(\mathrm{G}^{2} / \mathrm{Hz}\right)$ with $\mathrm{G}_{\mathrm{RMS}}=7.023$, considering equations 12 and 19 , the transmissibility ratio is $\mathrm{T}_{\mathrm{r}}=0.3243$. From Barry Slide Rule:

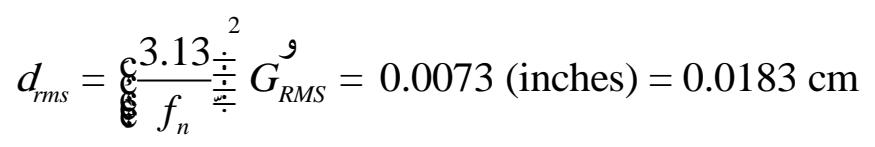

Because at $1 \delta$; the percentage of probability of actual deflection to snub is excessively high; i.e. more than $30 \%$. At $3 \delta$ the percentage of probability of actual deflection is $<0.3 \%$. This yields to $d_{\text {necessary }}=3 . d_{r m s}=3 * 0.0183=0.054898 \mathrm{~cm}$. 


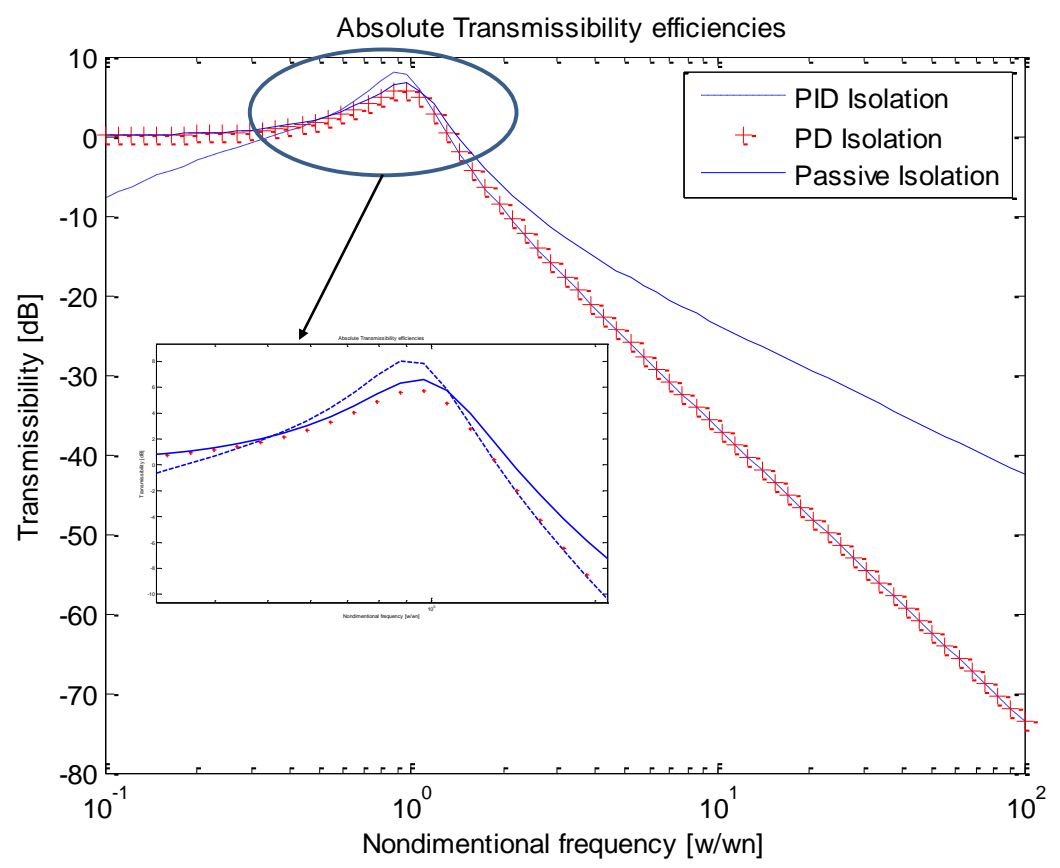

Fig. 13: Passive and active isolation results

Designing of PID controller and tuning of its gains are not simple step; different methods were discussed at previous work [8, 17-18]. In current work, the controlled system is designed using both PI and PID controllers based on an experimental data of passive vibration isolation system and vibration shaker test.

Time passive and active responses for the system due to random vibration excitation for active PI and PID isolation approaches are shown in figure 14.

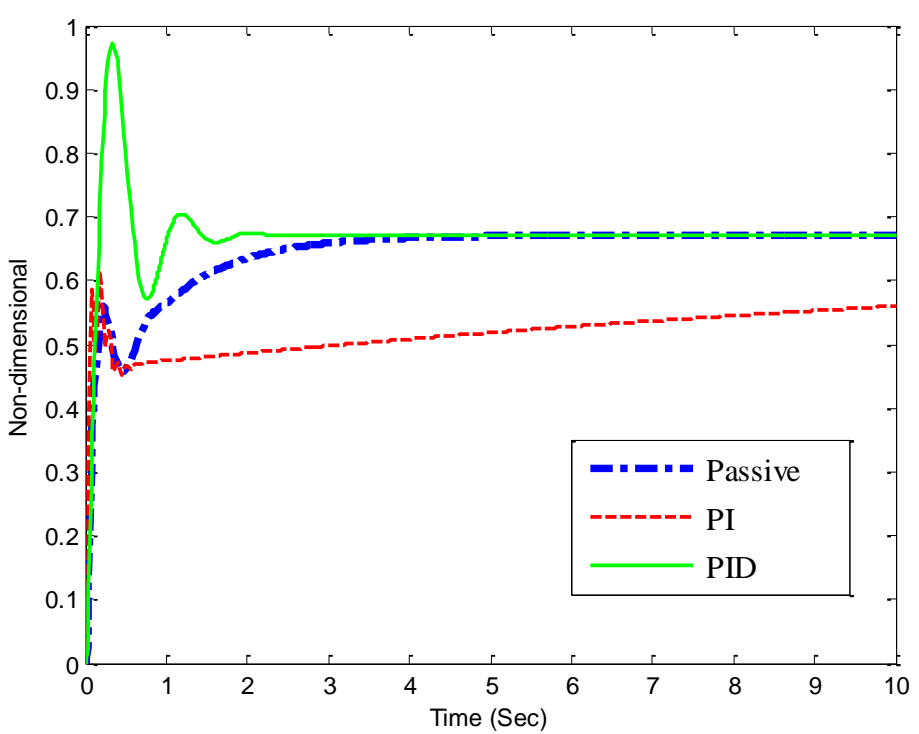

Fig. 14: time output response 


\section{Conclusion}

IMU is crucial equipment for navigation, guidance and control missions. Studying of flexibility environment using MSTMM provides an accurate analysis without truncation errors as considered in previous works. An active isolation system is modeled using PI and PID controllers based on an experimental data resulted from a vibration shaker test for a passive isolated IMU system. Current work provides protection and increment of the IMU response accuracy by mitigating effects of transferred disturbances due to the surrounded environment vibration and noise. Dilatation in studying controlled systems using MSTMM provides a possibility of incoming control applications because of the efficient dealing with the increased complexities of multi-rigid-flexible-body problems.

Acknowledgments: The research was supported by the Research Fund for the Doctoral Program of Higher Education of China (20113219110025).

\section{References}

[1] W. Thomson, Theory of Vibration with Applications, Second Edition, Prentice-Hall, New Jersey, 1981.

[2] Lee U. "Vibration analysis of one-dimensional structures using the spectral transfer matrix method." Engineering structures 22.6 (2000): 681-690.

[3] T. Irvine, Free Vibration of a Single-Degree-of-Freedom System, Vibration data Publications, 1999.

[4] Bokaian A. "Natural frequencies of beams under compressive axial loads."Journal of sound and vibration 126.1 (1988): 49-65.

[5] Ribeiro A, Silva J, Maia N. "On the generalisation of the transmissibility concept." Mechanical Systems and Signal Processing 14.1 (2000): 29-35.

[6] Maia N, Silva J, Ribeiro A. (2001). The transmissibility concept in multi-degreeoffreedom systems. Mechanical Systems and Signal Processing, 15(1), 129-137.

[7] Urgueira A P, Almeida R A, Maia N. "On the use of the transmissibility concept for the evaluation of frequency response functions", Mechanical Systems and Signal Processing 25.3 (2011): 940-951.

[8] Hossam Hendy, "Dynamic Modeling, Simulation and Control of a Navigation System with an IMU", PhD Nanjing Univ. of science and Tech., 2014.

[9] Shamsuzzoha M. "Closed-Loop PI/PID Controller Tuning for Stable and Integrating Process with Time Delay." Industrial \& Engineering Chemistry Research 52.36 (2013): 12973-12992.

[10] Rong B, Rui X T, Wang G P, et al. "Discrete time transfer matrix method for dynamics of multibody system with real-time control." Journal of Sound and Vibration 329.6 (2010): 627-643.

[11] Shamsuzzoha M, Lee M, Seki H. "Closed-loop PI/PID controller tuning for stable and unstable processes." American Control Conference (ACC), 2012. IEEE, 2012.

[12] $\mathrm{Wu} \mathrm{J} \mathrm{S}$, Chen $\mathrm{C}$ T. "A continuous-mass TMM for free vibration analysis of a nonuniform beam with various boundary conditions and carrying multiple concentrated elements." Journal of Sound and Vibration 311.3 (2008): 1420-1430

[13] Rui, et al; Advances in transfer matrix method of multibody system; ASME; 2009, DETC2009.

[14] Zhan, et al; Vibration control for launcher of multiple launch rocket system based on transfer matrix method of multibody system; Chinese Journal of Theoretical and Applied Mechanics, May 2010 
[15] K. J., Astrom, T. Hagglund, C. C. Hang, and W. K. Ho, "Automatic tuning and adaptation for PID controllers-A survey," IFACJ Control Eng Practice, 1(4): 699-714, 1993.

[16] Jing-Chung Shen, New Tuning Method for PID Controller, Mexico; Control Applications IEEE. 41(4): 473-484, 2002.

[17] Hossam Hendy et al, Controller Parameters Tuning Based on Transfer Matrix Method for Multibody Systems, Journal of Advances in Mechanical Engineering, 2013.

[18] Bestle, Rui, Application of the Transfer Matrix Method to Control Problems; Thematic, Croatia 2013. 